\title{
Radiation of a charge moving along the boundary of dielectric prism
}

\author{
A. V. Tyukhtin, ${ }^{*}$ V. V. Vorobev, and S. N. Galyamin \\ Saint-Petersburg State University, 7/9 Universitetskaya naberezhnaya, St. Petersburg 199034, Russia \\ E. S. Belonogaya \\ Saint-Petersburg State University, 7/9 Universitetskaya naberezhnaya, St. Petersburg 199034, Russia \\ and Saint-Petersburg State Electrotechnical University "LETI”, 5 Professora Popova, \\ St. Petersburg 197376, Russia
}

(Received 13 November 2018; published 2 January 2019)

\begin{abstract}
Radiation generated by a charge moving along one of the prism borders is analyzed. The size of the object is assumed to be much larger than the wavelengths under consideration. We calculate the wave field outside the target using the "aperture method" which is applicable for any distances from the prism. We obtain the particular form of Stratton-Chu formulas for the case of the prism and develop an algorithm for the calculation of the radiation field outside the prism. Typical numerical results are demonstrated and discussed.
\end{abstract}

DOI: 10.1103/PhysRevAccelBeams.22.012802

\section{INTRODUCTION}

The radiation of charged particles in the presence of dielectric objects with a complicated form is of essential interest for applications in accelerator and beam physics [1-5]. As an example, one can point out a new method of noninvasive bunch diagnostics where Cherenkov radiation (frequently also referred to as Cherenkov-diffraction radiation) outside a dielectric object should be registered [2,5]. As a rule, the complex geometry of the object does not allow obtaining the rigorous solution of the problem. At the same time, the size of the target is frequently much larger compared to the wavelength under consideration, and this fact complicates considerably computer simulations. On the contrary, from a theoretical point of view, it gives us an obvious small parameter of the problem (wavelength to target size ratio) and allows the development of efficient approximate methods of analysis.

In previous papers, we have offered two methods for the solution of such problems which can be named "the ray-optics method" and "the aperture method" [6-11]. Both methods are valid for objects which are much larger than the wavelength under consideration, $\lambda$.

The first step is the same for both methods: We should solve a certain "etalon problem" which does not take into account "external" boundaries of the target. For example,

\footnotetext{
*a.tyuhtin@spbu.ru, tyukhtin@bk.ru
}

Published by the American Physical Society under the terms of the Creative Commons Attribution 4.0 International license. Further distribution of this work must maintain attribution to the author(s) and the published article's title, journal citation, and DOI. if a charge moves along one of the flat object boundaries, then the corresponding etalon problem is the following: the interaction of a charge with a semi-infinite medium. The field obtained in this problem (namely, the field of Cherenkov radiation) can be named an "incident" field.

At the second step, we determine the part of the external boundary which is illuminated by Cherenkov radiation (further it is called an "aperture") and use the fact that the object is large in comparison with the wavelengths under consideration. We obtain the asymptotic of the incident field and present it as a sum of waves of two polarizations ("vertical" and "horizontal" with respect to the aperture). The field at the external surface of the aperture is calculated using Snell's and Fresnel's laws.

The last steps of the two methods are different. The rayoptics method uses the ray optic laws for the calculation of the wave field outside the object [6,7]. However, this technique has essential additional limitations. The distance $R$ from the aperture to the observation point should be "not very large"; i.e., the so-called wave parameter $D$ should be small: $D \sim \lambda R / \Sigma \ll 1$, where $\Sigma$ is the aperture area. Moreover, the observation point cannot be close to the focuses and caustics.

However, these conditions are often not met. Therefore, we have developed a more general "aperture method" which is valid for an observation point with arbitrary wave parameter $D$ including a Fraunhofer (far-field) area (where $D \gg 1$ ) and neighborhoods of focuses and caustics [8-11]. At the final step of this technique, we calculate the field outside the target using Stratton-Chu formulas ("aperture integrals"). These formulas allow determining the field in the surrounding space if tangential components of electric and magnetic fields on the aperture are known. 
Naturally, the aperture method is approximate. In particular, it does not take into account any interior rereflections of waves inside the object. Therefore, we have verified this method for an axially symmetrical object- the cone with a vacuum channel where the charge moves [10]. Analytical results have been compared with numerical results obtained via simulations in COMSOL Multiphysics. In the issue, we have concluded that the aperture method can be applied even for relatively small objects with the size of several wavelengths. It is important as well that the accuracy of calculations increases with an increase in the distance from the aperture [10].

In this paper, we consider another promising target in the shape of a prism. This object is actively used not only in optics but also in accelerator and beam physics. For instance, such a target is offered to be applied in the new noninvasive method of bunch diagnostics $[2,5]$. Note that earlier we have studied the charge radiation in the presence of such an object [7] but only in the region of ray-optics applicability. Meanwhile, the experimental practice usually needs knowledge of the radiation characteristics in the far-field (Fraunhofer) area, where the ray-optics technique is not applicable. The present paper is devoted mainly to this problem.

\section{APERTURE INTEGRALS}

Aperture integrals (Stratton-Chu formulas) for the Fourier transform of an electric field [12] can be written in the following general form (we use the Gaussian system of units):

$$
\begin{aligned}
\vec{E}(\vec{R})= & \vec{E}^{(h)}(\vec{R})+\vec{E}^{(e)}(\vec{R}), \\
\vec{E}^{(h)}(\vec{R})= & \frac{i k}{4 \pi} \int_{\Sigma}\left\{\left[\vec{n}^{\prime} \times \vec{H}\left(\vec{R}^{\prime}\right)\right] G\left(\left|\Delta \vec{R}^{\prime}\right|\right)\right. \\
& \left.+\frac{1}{k^{2}}\left(\left[\vec{n}^{\prime} \times \vec{H}\left(\vec{R}^{\prime}\right)\right] \cdot \nabla^{\prime}\right) \nabla^{\prime} G\left(\left|\Delta \vec{R}^{\prime}\right|\right)\right\} d \Sigma^{\prime}, \\
\vec{E}^{(e)}(\vec{R})= & \frac{1}{4 \pi} \int_{\Sigma}\left\{\left[\vec{n}^{\prime} \times \vec{E}\left(\vec{R}^{\prime}\right)\right] \times \nabla^{\prime} G\left(\left|\Delta \vec{R}^{\prime}\right|\right)\right\} d \Sigma^{\prime},
\end{aligned}
$$

where $\Sigma$ is an aperture area, $\Delta \vec{R}^{\prime}=\vec{R}-\vec{R}^{\prime}, \vec{E}\left(\vec{R}^{\prime}\right)$ and $\vec{H}\left(\vec{R}^{\prime}\right)$ are Fourier transforms of the field components on the aperture, $k=\omega / c$ is a wave number of the outer space (vacuum), $\vec{n}^{\prime}$ is a unit external normal to the aperture in the point $\vec{R}^{\prime}, G(R)=\exp (i k R) / R$ is a Green function of the Helmholtz equation, and $\nabla^{\prime}$ is a gradient: $\nabla^{\prime}=\vec{e}_{x} \partial / \partial x^{\prime}+$ $\vec{e}_{y} \partial / \partial y^{\prime}+\vec{e}_{z} \partial / \partial z^{\prime}$. Analogous formulas are known for the magnetic field as well; however, we do not write them here, because we are interested in "a wave zone" where the distance from the aperture to the observation point is assumed to be much more than the wavelength. In this zone, the field is close to the plane wave; therefore, vectors $\vec{E}, \vec{H}$, and $\vec{k}$ form a right orthogonal triad and $|\vec{E}| \approx|\vec{H}|$.

The observation point is often located far from the target, in the region where the so-called wave parameter is large:

$$
D \sim \lambda R / \Sigma \sim \lambda R / d^{2} \gg 1,
$$

where $\lambda$ is a wavelength under consideration, $R$ is a distance from the target to the observation point, and $\Sigma$ is a square of an aperture (we assume that $\Sigma \sim d^{2}$, where $d$ is a typical linear size of the aperture). This region is usually referred to as the Fraunhofer area. The general formulas (1) can be essentially simplified in this area.

Note that the condition (2) results in the inequality

$$
R \gg d \cdot(d / \lambda) \gg d,
$$

because $d \gg \lambda$ in the problem under consideration. Using the inequalities (2) and (3) and taking into account that $\left|\vec{R}^{\prime}\right| \sim d$, one can apply the following approximation in the formulas (1):

$$
G\left(\left|\vec{R}-\vec{R}^{\prime}\right|\right) \approx \frac{\exp \left(i k R-i k \vec{R} \vec{R}^{\prime} / R\right)}{R} .
$$

As a result, we obtain the following formulas in the Fraunhofer area:

$$
\begin{aligned}
\vec{E}^{(h)} \approx & \frac{i k e^{i k R}}{4 \pi R} \int_{\Sigma}\left\{\left[\vec{n}^{\prime} \times \vec{H}\left(\vec{R}^{\prime}\right)\right]\right. \\
& \left.-\vec{e}_{R}\left(\vec{e}_{R} \cdot\left[\vec{n}^{\prime} \times \vec{H}\left(\vec{R}^{\prime}\right)\right]\right)\right\} e^{-i k \vec{e}_{R} \vec{R}^{\prime}} d \Sigma^{\prime}, \\
\vec{E}^{(e)} \approx & \frac{i k e^{i k R}}{4 \pi R} \int_{\Sigma}\left\{\vec{e}_{R} \times\left[\vec{n}^{\prime} \times \vec{E}\left(\vec{R}^{\prime}\right)\right]\right\} e^{-i k \vec{e}_{R} \vec{R}^{\prime}} d \Sigma^{\prime},
\end{aligned}
$$

where $\vec{e}_{R}=\vec{R} / R$.

\section{APERTURE INTEGRALS FOR PRISMATIC OBJECT}

The considered object is a dielectric prism made of material possessing permittivity $\varepsilon$, permeability $\mu$, and negligible conductivity. The charge $q$ moves with constant velocity $\vec{V}=c \beta \vec{e}_{z}$ along the $z$ axis, which is parallel to one of the prism boundaries (Fig. 1). The distance from the charge trajectory to the nearest boundary of the prism is $a$ (this value can be arbitrary). The charge velocity is larger than the "Cherenkov threshold" $(\beta>1 / \sqrt{\varepsilon \mu})$; therefore, Cherenkov radiation is generated in the prism material.

For definiteness, we deal with a point charge having the charge density $\rho=q \delta(x) \delta(y) \delta(z-V t)$, where $\delta(z-V t)$ is a Dirac delta function. However, the results obtained below

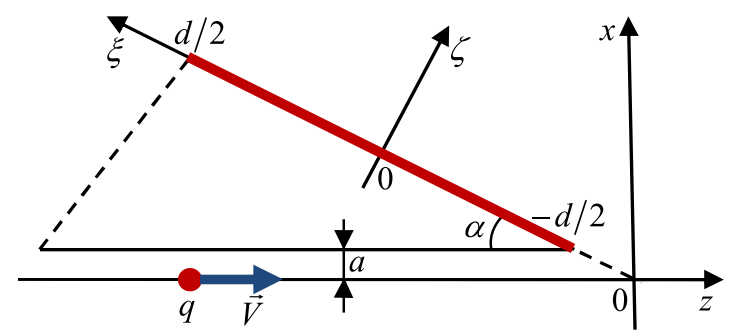

FIG. 1. Cross section of the prism and the charge moving along the border. The aperture is shown by the heavy red line. 
can be easily generalized for the case of a thin bunch with finite length, because we consider Fourier transforms of the field components.

Note that the form of the "back" border of the target (dotted line in Fig. 1) is not important. It is important only that the external border (the aperture with the size $d \gg \lambda$ shown by the bold red line in Fig. 1) is illuminated by Cherenkov radiation.

For convenience, we introduce the coordinate system $\xi, \eta$, $\zeta$ associated with the aperture (Fig. 1): the axes $\xi$ and $\eta=y$ are on the aperture, the axis $\zeta$ is orthogonal to it, and the point $\xi=\eta=\zeta=0$ is the aperture center. We assume that the aperture is a rectangle with sides $d$ (along $\xi$ ) and $b$ (along $\eta$ ): it is determined by the inequalities $|\xi| \leq d / 2$ and $|\eta| \leq b / 2$.

We will consider the distances which are essentially larger than the wavelength $(k \zeta \gg 1$ and, correspondingly, $k R \gg 1$ as well). Using this condition, we can differentiate only the function $\exp \left(i k\left|\vec{R}-\vec{R}^{\prime}\right|\right)$ in (1), considering the function $1 /\left|\vec{R}-\vec{R}^{\prime}\right|$ to be constant. Taking into account this fact, for the geometry of the prism one can obtain from (1) the following expressions:

$$
\begin{gathered}
\left\{\begin{array}{c}
E_{\xi}^{(h)}(\vec{R}) \\
E_{\eta}^{(h)}(\vec{R}) \\
E_{\zeta}^{(h)}(\vec{R})
\end{array}\right\}=\frac{i k}{4 \pi} \int_{-d / 2}^{d / 2} d \xi^{\prime} \int_{-b / 2}^{b / 2} d \eta^{\prime} \frac{G\left(\left|\Delta \vec{R}^{\prime}\right|\right)}{\left(\Delta \vec{R}^{\prime}\right)^{2}} \times\left\{\begin{array}{l}
-\left[\left(\Delta \eta^{\prime}\right)^{2}+\zeta^{2}\right] H_{\eta}\left(\vec{R}^{\prime}\right)-\Delta \xi^{\prime} \Delta \eta^{\prime} H_{\xi}\left(\vec{R}^{\prime}\right) \\
{\left[\left(\Delta \xi^{\prime}\right)^{2}+\zeta^{2}\right] H_{\xi}\left(\vec{R}^{\prime}\right)+\Delta \xi^{\prime} \Delta \eta^{\prime} H_{\eta}\left(\vec{R}^{\prime}\right)} \\
-\zeta \Delta \eta^{\prime} H_{\xi}\left(\vec{R}^{\prime}\right)+\zeta \Delta \xi^{\prime} H_{\eta}\left(\vec{R}^{\prime}\right)
\end{array}\right\}, \\
\left\{\begin{array}{l}
E_{\xi}^{(e)}(\vec{R}) \\
E_{\eta}^{(e)}(\vec{R}) \\
E_{\zeta}^{(e)}(\vec{R})
\end{array}\right\}=\frac{i k}{4 \pi} \int_{-d / 2}^{d / 2} d \xi^{\prime} \int_{-b / 2}^{b / 2} d \eta^{\prime} \frac{G\left(\left|\Delta \vec{R}^{\prime}\right|\right)}{\left|\Delta \vec{R}^{\prime}\right|} \times\left\{\begin{array}{l}
-\zeta E_{\xi}\left(\vec{R}^{\prime}\right) \\
-\zeta E_{\eta}\left(\vec{R}^{\prime}\right) \\
\Delta \xi^{\prime} E_{\xi}\left(\vec{R}^{\prime}\right)+\Delta \eta^{\prime} E_{\eta}\left(\vec{R}^{\prime}\right)
\end{array}\right\},
\end{gathered}
$$

where $\vec{R}^{\prime}=\left(\xi^{\prime}, \eta^{\prime}, 0\right), \Delta \xi^{\prime}=\xi-\xi^{\prime}$, and $\Delta \eta^{\prime}=\eta-\eta^{\prime}$. These formulas are correct in a wide range of distances limited only by the condition $k \zeta \gg 1$. They can be applied for a ray-optics area $[D=\lambda \zeta /(b d) \ll 1]$, Fresnel area $(D \sim 1)$, and Fraunhofer area $(D \gg 1)$.

In the far-field (Fraunhofer) area, the field can be calculated using formulas (5). One can show that for the case of the prism these formulas can be written in the following form:

$$
\begin{gathered}
\left\{\begin{array}{c}
E_{\xi}^{(h)} \\
E_{\eta}^{(h)} \\
E_{\zeta}^{(h)}
\end{array}\right\} \approx \frac{i k \exp (i k R)}{4 \pi R} \int_{\Sigma} \exp \left(-\frac{i k}{R}\left(\xi \xi^{\prime}+\eta \eta^{\prime}\right)\right) \times\left\{\begin{array}{l}
-H_{\eta}\left(\vec{R}^{\prime}\right)+\xi R^{-2}\left[\xi H_{\eta}\left(\vec{R}^{\prime}\right)-\eta H_{\xi}\left(\vec{R}^{\prime}\right)\right] \\
H_{\xi}\left(\vec{R}^{\prime}\right)+\eta R^{-2}\left[\xi H_{\eta}\left(\vec{R}^{\prime}\right)-\eta H_{\xi}\left(\vec{R}^{\prime}\right)\right] \\
\zeta R^{-2}\left[\xi H_{\eta}\left(\vec{R}^{\prime}\right)-\eta H_{\xi}\left(\vec{R}^{\prime}\right)\right]
\end{array}\right\} d \Sigma^{\prime}, \\
\left\{\begin{array}{l}
E_{\xi}^{(e)} \\
E_{\eta}^{(e)} \\
E_{\zeta}^{(e)}
\end{array}\right\} \approx \frac{i k \exp (i k R)}{4 \pi R^{2}} \int_{\Sigma} \exp \left(-\frac{i k}{R}\left(\xi \xi^{\prime}+\eta \eta^{\prime}\right)\right) \times\left\{\begin{array}{l}
-\zeta E_{\xi}\left(\vec{R}^{\prime}\right) \\
-\zeta E_{\eta}\left(\vec{R}^{\prime}\right) \\
\xi E_{\xi}\left(\vec{R}^{\prime}\right)+\eta E_{\eta}\left(\vec{R}^{\prime}\right)
\end{array}\right\} d \Sigma^{\prime} .
\end{gathered}
$$

Naturally, the same result can be found from (6) as well.

In the Fraunhofer area, it is convenient to use the spherical coordinates $R, \Theta$, $\Phi$ which are associated with coordinates $\xi, \eta, \zeta$ :

$$
\xi=R \sin \Theta \cos \Phi, \quad \eta=R \sin \Theta \sin \Phi, \quad \zeta=R \cos \Theta .
$$

Using these coordinates, one can obtain from (7) the following expressions:

$$
\begin{aligned}
& E_{R} \approx 0, \\
&\left\{\begin{array}{l}
E_{\Theta} \\
E_{\Phi}
\end{array}\right\} \approx \frac{i k \exp (i k R)}{4 \pi R} \int_{\Sigma} e^{-i k\left(\xi^{\prime} \cos \Phi+\eta^{\prime} \sin \Phi\right) \sin \Theta} \times\left\{\begin{array}{c}
-E_{\xi} \cos \Phi-E_{\eta} \sin \Phi+\left[H_{\xi} \sin \Phi-H_{\eta} \cos \Phi\right] \cos \Theta \\
H_{\xi} \cos \Phi+H_{\eta} \sin \Phi+\left[E_{\xi} \sin \Phi-E_{\eta} \cos \Phi\right] \cos \Theta
\end{array}\right\} d \Sigma^{\prime},
\end{aligned}
$$


where $E_{\xi, \eta}=E_{\xi, \eta}\left(\vec{R}^{\prime}\right)$ and $H_{\xi, \eta}=H_{\xi, \eta}\left(\vec{R}^{\prime}\right)$. The first equality means that the wave is transversal (as expected). The magnetic field value is equal to the electric field value, and vectors $\vec{E}, \vec{H}$, and $\vec{k}^{(t)}$ form the right orthogonal triad.

\section{THE FIELD ON THE PRISM APERTURE}

In order to apply the formulas (6) or (9), we have to obtain the tangential components $E_{\xi, \eta}$ and $H_{\xi, \eta}$ on the aperture. According to our method, we first find the incident field $\vec{E}^{(i)}, \vec{H}^{(i)}$ assuming that the external boundaries of the object are absent. In the case under consideration (see Fig. 1), we exclude the back boundary, the aperture, and prolongate target material in the area $x>a$, $-\infty<z<+\infty$. In the issue, we obtain the problem for Cherenkov radiation of a charge moving along the plane interface between a vacuum and a medium. The solution of such a problem is known [7,13]. It should be underlined that we need to know the field in the far-field area, because the main part of the aperture is placed far from the charge trajectory.

$$
\begin{aligned}
& h_{x}=k \beta^{-1} w_{y}-k_{y} w_{z}, \quad h_{y}=k \beta^{-1} w_{x}-g_{m} w_{z}, \quad h_{z}=g_{m} w_{y}-k_{y} w_{x}, \quad k_{y}=\frac{s y}{\sqrt{(x-a)^{2}+y^{2}}}, \\
& w_{x}=\frac{-2 g_{v}}{k\left(\varepsilon g_{v}+g_{m}\right)}, \quad w_{y}=\frac{-2 k_{y}\left(g_{v}+\mu g_{m}\right)}{k\left(\varepsilon g_{v}+g_{m}\right)\left(\mu g_{v}+g_{m}\right)}, \quad w_{z}=\frac{2 \mu \beta}{\mu g_{v}+g_{m}}-\frac{2}{\beta\left(\varepsilon g_{v}+g_{m}\right)}, \\
& g_{v}=i \sqrt{\kappa^{2}+k_{y}^{2}}, \quad g_{m}=\sqrt{s^{2}-k_{y}^{2}}, \quad k=\frac{\omega}{c}, \\
& s=k \beta^{-1} \sqrt{\varepsilon \mu \beta^{2}-1}, \quad \kappa=k \beta^{-1} \sqrt{1-\beta^{2}}, \quad S=(x-a) g_{m}+k_{y} y+k \beta^{-1} z .
\end{aligned}
$$

The coordinates $\xi, \eta, \zeta$ relate to $x, y, z$ in the following way:

$$
\begin{aligned}
\xi= & \left(x-x_{c}\right) \sin \alpha-\left(z-z_{c}\right) \cos \alpha, \\
\eta= & y, \\
\zeta= & \left(x-x_{c}\right) \cos \alpha+\left(z-z_{c}\right) \sin \alpha, \\
& x=x_{c}+\xi \sin \alpha+\zeta \cos \alpha, \\
& y=\eta, \\
& z=z_{c}-\xi \cos \alpha+\zeta \sin \alpha,
\end{aligned}
$$

where $x_{c}=a+\frac{d}{2} \sin \alpha, \quad z_{c}=-a \cot \alpha-\frac{d}{2} \cos \alpha \quad$ is the center of the aperture.

Consider some point $\xi^{\prime}, \eta^{\prime}, \zeta^{\prime}=0$ on the aperture. The wave vector $\vec{k}^{(i)}$ of the incident wave falling onto this point has the following components:
After some transformations of formulas from Refs. [7,13], one can write the incident field in the following form:

$$
\begin{aligned}
\left\{\begin{array}{l}
H_{x}^{(i)} \\
H_{y}^{(i)} \\
H_{z}^{(i)} \\
E_{x}^{(i)} \\
E_{y}^{(i)} \\
E_{z}^{(i)}
\end{array}\right\}= & \left\{\begin{array}{l}
-h_{x} \\
-h_{y} \\
-h_{z} \\
\varepsilon^{-1}\left(k^{-1} k_{y} h_{z}-\beta^{-1} h_{y}\right) \\
\varepsilon^{-1}\left(\beta^{-1} h_{x}-k^{-1} g_{m} h_{z}\right) \\
\varepsilon^{-1} k^{-1}\left(g_{m} h_{y}-k_{y} h_{x}\right)
\end{array}\right\} \\
& \times \frac{q \sqrt{s}(x-a) \exp \left(-a \sqrt{\kappa^{2}+k_{y}^{2}}+i S-i \frac{\pi}{4}\right)}{\sqrt{2 \pi} c \beta \mu\left[(x-a)^{2}+y^{2}\right]^{3 / 4}},
\end{aligned}
$$

where

$$
\begin{aligned}
& k_{x}^{(i)}=k \sqrt{\varepsilon \mu} \sin \theta_{p} \cos \varphi^{\prime}, \\
& k_{y}^{(i)}=k \sqrt{\varepsilon \mu} \sin \theta_{p} \sin \varphi^{\prime}, \\
& k_{z}^{(i)}=k \sqrt{\varepsilon \mu} \cos \theta_{p},
\end{aligned}
$$

where $\theta_{p}=\arccos \left[(\sqrt{\varepsilon \mu} \beta)^{-1}\right]$ is the Cherenkov angle and $\varphi^{\prime}$ is a cylindrical coordinate of a point on the aperture: $\cos \varphi^{\prime}=x^{\prime} / \sqrt{x^{\prime 2}+y^{\prime 2}}, \sin \varphi^{\prime}=y^{\prime} / \sqrt{x^{\prime 2}+y^{\prime 2}}$. The angle of incidence $\theta_{i}$ (with respect to the normal $\vec{e}_{\zeta}$ ) is determined by the relation

$$
\begin{aligned}
\cos \theta_{i} & =\left(\vec{e}_{\zeta}, \vec{k}^{(i)} / k^{(i)}\right) \\
& =\cos \alpha \sin \theta_{p} \cos \varphi^{\prime}+\sin \alpha \cos \theta_{p} .
\end{aligned}
$$

The angle of refraction is determined by Snell's law:

$$
\sin \theta_{t}=\sqrt{\varepsilon \mu} \sin \theta_{i}
$$

Further we should separate the incident waves of horizontal $(h)$ and vertical $(v)$ polarizations (with respect 
to the incidence plane which is formed by the normal $\vec{e}_{\zeta}$ and the wave vector $\vec{k}^{(i)}$ ). The incident wave of horizontal polarization has the electric field component

$$
\vec{E}_{h}^{(i)}=E_{h \psi}^{(i)} \vec{\psi} / \psi=\left(\vec{E}^{(i)}, \vec{\psi}\right) \vec{\psi} / \psi^{2}
$$

(a)

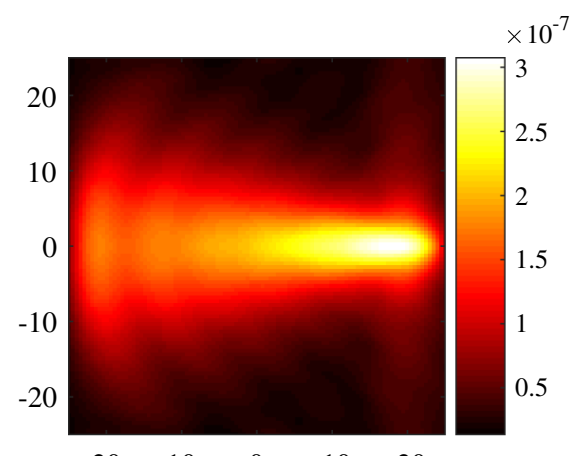

(b)
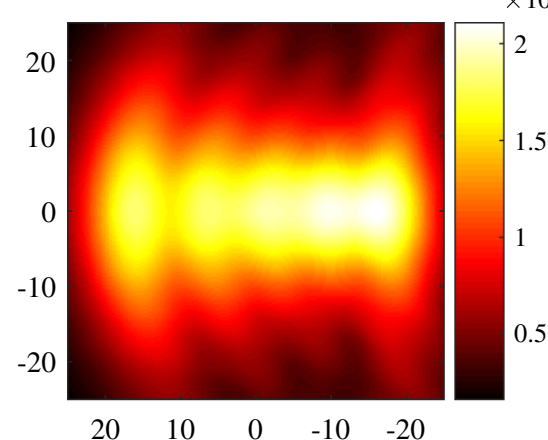

(c)
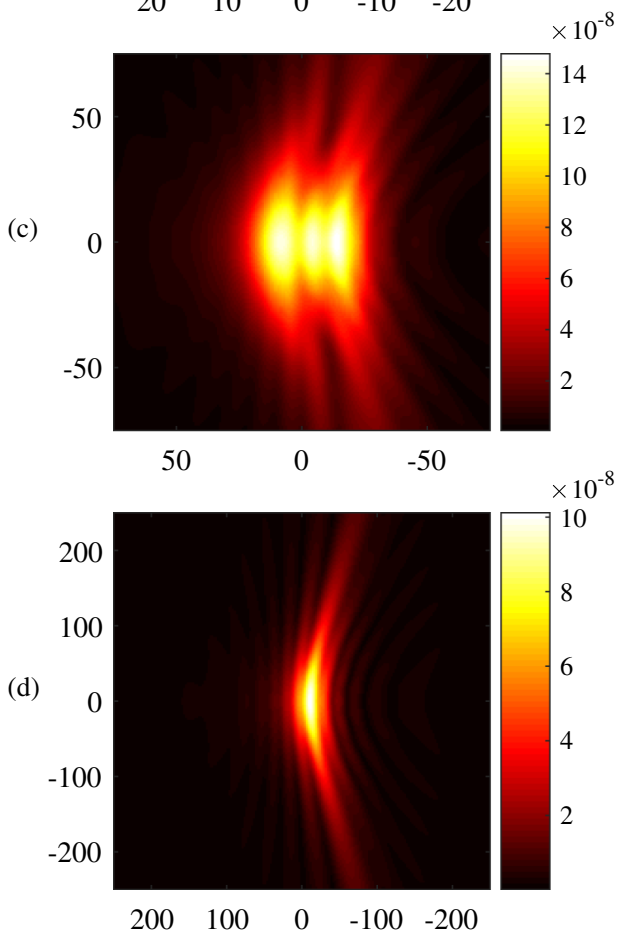

and the incident wave of vertical polarization has the magnetic field component

$$
\vec{H}_{v}^{(i)}=H_{v \psi}^{(i)} \vec{\psi} / \psi=\left(\vec{H}^{(i)}, \vec{\psi}\right) \vec{\psi} / \psi^{2}
$$
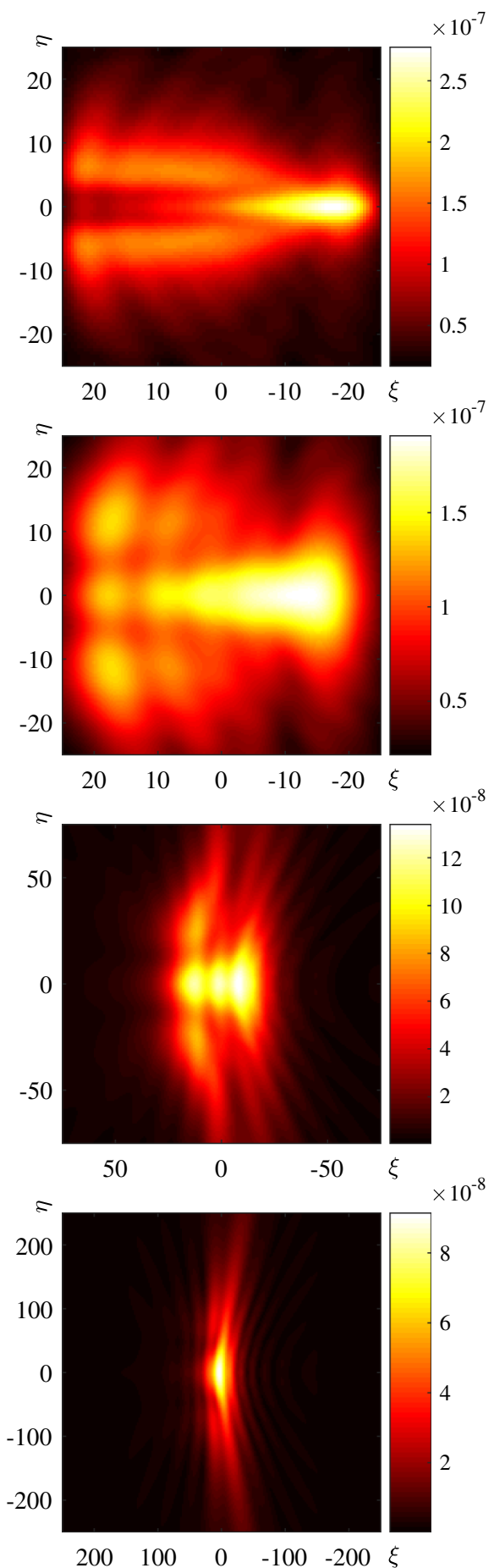

FIG. 2. Electric field magnitude $|\vec{E}|(\mathrm{V} \cdot \mathrm{s} / \mathrm{m})$ in the plane $\xi, \eta$ (parallel to the aperture) for the following parameters: $\varepsilon=4, \mu=1$, $d=b=50 k^{-1}, a k=1, \alpha=30^{0}, q=1 \mathrm{nC} ; \beta=0.9$ (left column) and $\beta=0.999$ (right column); (a) $\zeta=2 k^{-1}$, (b) $\zeta=10 k^{-1}$, (c) $\zeta=30 k^{-1}$, and (d) $\zeta=100 k^{-1}$. 
Here $\vec{\psi}$ is a vector which is orthogonal to the plane of incidence: $\vec{\psi}=\left[\vec{e}_{\zeta}, \vec{k}^{(i)}\right]$. One can obtain that its components can be written in the form

$$
\begin{aligned}
& \psi_{x}=-\sin \alpha \sin \theta_{p} \sin \varphi^{\prime}, \\
& \psi_{y}=\sin \alpha \sin \theta_{p} \cos \varphi^{\prime}-\cos \alpha \cos \theta_{p}, \\
& \psi_{z}=\cos \alpha \sin \theta_{p} \sin \varphi^{\prime} .
\end{aligned}
$$

The field on the external border of the prism is determined with the help of Fresnel coefficients. The waves of horizontal and vertical polarization have the following components, respectively:

(a)

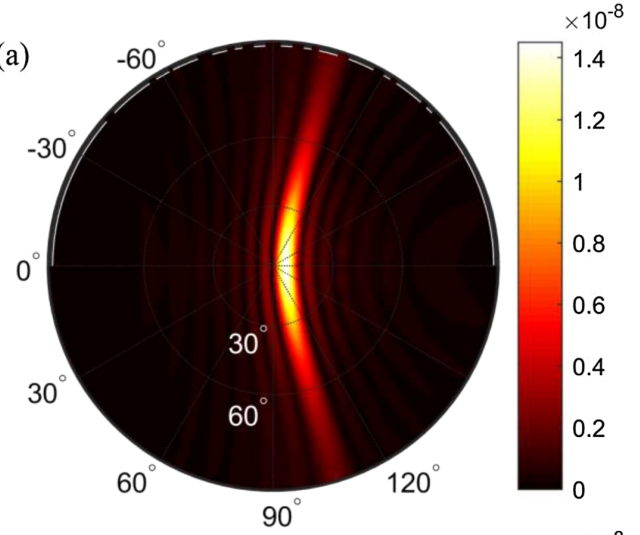

90

(b)
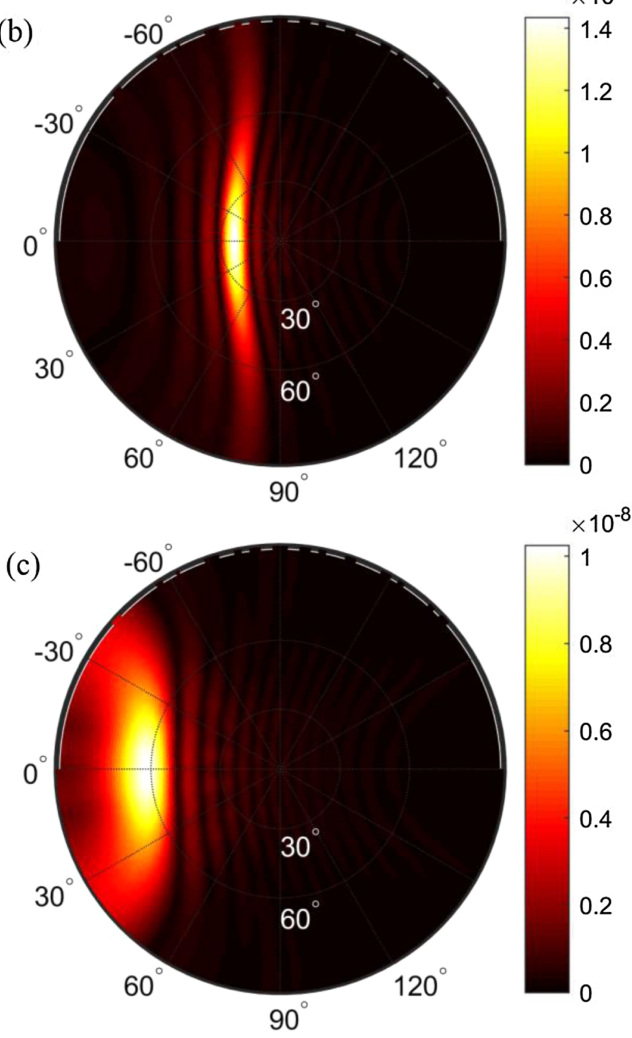

$$
\begin{array}{rlrl}
\vec{E}_{h} & =T_{h} E_{h \psi}^{(i)} \vec{\psi} / \psi, & \vec{H}_{h}=\left[\vec{k}^{(t)} / k, \vec{E}_{h}\right], \\
\vec{H}_{v}=T_{v} H_{v \psi}^{(i)} \vec{\psi} / \psi, & \vec{E}_{v}=-\left[\vec{k}^{(t)} / k, \vec{H}_{v}\right],
\end{array}
$$

where

$T_{h}=\frac{2 \cos \theta_{i}}{\cos \theta_{i}+Z \cos \theta_{t}}, \quad T_{v}=\frac{2 Z \cos \theta_{i}}{Z \cos \theta_{i}+\cos \theta_{t}}$,

and $Z=\sqrt{\mu / \varepsilon}$ is an impedance of the prism material.

After some transformations, one can obtain the following expressions for the tangential components of the field at arbitrary point $\vec{R}^{\prime}=\left(\xi^{\prime}, \eta^{\prime}, 0\right)$ on the aperture:
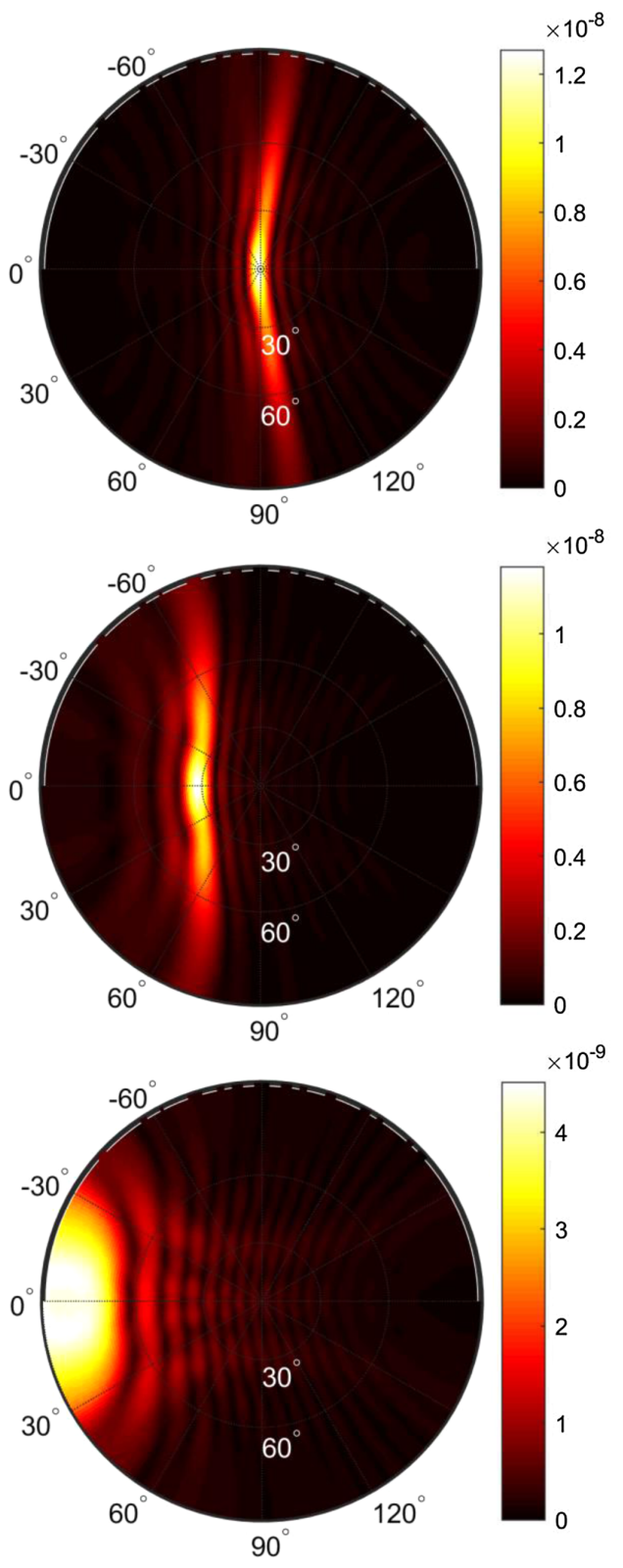

FIG. 3. Electric field magnitude $|\vec{E}|(\mathrm{V} \cdot \mathrm{s} / \mathrm{m})$ in the far-field zone at $R=1000 k^{-1}$. Spherical coordinate system (8) is used, the radial axis represents the $\Theta$ angle, and the polar axis is the $\Phi$ angle. Problem parameters: $q=1 \mathrm{nC}, \varepsilon=4, \mu=1, d=b=50 k^{-1}, a k=1$; (a) $\alpha=30^{\circ}$, (b) $\alpha=45^{\circ}$, (c) $\alpha=60^{\circ} ; \beta=0.9$ (left column) and $\beta=0.999$ (right column). 


$$
\begin{aligned}
& E_{\xi}=-U \cdot E_{h \psi}+\cos \theta_{t} \cdot V \cdot H_{v \psi}, \\
& E_{\eta}=V \cdot E_{h \psi}+\cos \theta_{t} \cdot U \cdot H_{v \psi}, \\
& H_{\xi}=-\cos \theta_{t} \cdot V \cdot E_{h \psi}-U \cdot H_{v \psi}, \\
& H_{\eta}=-\cos \theta_{t} \cdot U \cdot E_{h \psi}+V \cdot H_{v \psi},
\end{aligned}
$$

where

$$
\begin{aligned}
U & =|\vec{\psi}|^{-1} \sin \theta_{p} \sin \varphi^{\prime}, \\
V & =|\vec{\psi}|^{-1}\left(\sin \alpha \sin \theta_{p} \cos \varphi^{\prime}-\cos \alpha \cos \theta_{p}\right) .
\end{aligned}
$$

Thus, we have written the field components on the prism surface in the form which is convenient for use in expressions (6) or (7).

\section{NUMERICAL RESULTS}

Usually, one characterizes the radiation with the help of energetic characteristics. The energy flow density is $\vec{S}=\frac{c}{4 \pi}[\overrightarrow{\mathbf{E}}, \overrightarrow{\mathbf{H}}]$, where $\{\overrightarrow{\mathbf{E}}, \overrightarrow{\mathbf{H}}\}=\int_{-\infty}^{\infty}\{\vec{E}, \vec{H}\} e^{-i \omega t} d \omega$. After some transformations, one can obtain that $\vec{S}=$ $\vec{e}_{R} \int_{0}^{\infty} \sigma d \omega$, where $\sigma=c|\vec{E}|^{2}=c\left|\vec{E}_{\Theta}+\vec{E}_{\Phi}\right|^{2}$ is a spectral density of the Pointing vector [7]. For convenience, we will give the plots for the module of Fourier transform of the electric field, that is, $|\vec{E}|=\sqrt{\sigma / c}$.

Some numerical results are shown in Figs. 2 and 3. Figure 2 shows the module of Fourier transform of an electric field in the plane which is parallel to the aperture for two different velocities of the charge $(\beta=0.9$ and $\beta=0.999$ ). In these plots, the horizontal axis is $\xi$, and the vertical axis is $\eta$. The top row corresponds to a relatively small distance from the aperture; below, this distance increases. One can see how the field distribution changes with an increase in the distance from the aperture. In particular, if the charge velocity is ultrarelativistic, then we have a complex picture with additional maximums outside the symmetry axis when the distance $\zeta$ is not very large (right column). For the lesser velocities, the picture is simpler: there is the single maximum on the symmetry axis (left column). This difference relates to the peculiarities of the incident field: if the charge velocity is large enough, then the radiation in the prism material has maximums which are not at the symmetry axis [13].

Note that such complex pictures do not take place in the far-field area, where diffraction "spreading" of the field results in similar plots for different velocities (bottom row in Fig. 2).

Figure 3 demonstrates the value $|\vec{E}|$ in the far-field area (note that the frequency-angular density of the radiation power is $\frac{d^{2} W}{d \omega d \Omega}=R^{2} \sigma=c R^{2}|\vec{E}|^{2}$ ). In these plots, spherical coordinates $\Theta, \Phi$ [see (8)] are used; the radial axis represents the angle $\Theta$, and the polar axis is the angle $\Phi$. We consider three prisms having different angles $\alpha$. As one can see, the radiation maximum lies in the plane $\eta=0$ and deviates with the angle $\alpha$. Calculations show that the direction of the radiation maximum coincides with the direction of the refracted Cherenkov ray (16).

\section{CONCLUSION}

We have analyzed the radiation from the charge which moves along one of the prism boundaries. The aperture method applied here uses the solution of a certain etalon problem (without external borders of the object), ray-optics laws on the aperture, and Stratton-Chu formulas for the field in the outer area. This method is applicable for any distances from the target including the far-field (Fraunhofer) area.

We have obtained the particular form of Stratton-Chu formulas for the prism (for arbitrary distances and, separately, for the far-field area). The algorithm of calculation of the radiation field outside the prism has been constructed. Typical numerical results show, in particular, that for an ultrarelativistic charge the field distribution can be very complicated at small enough distances from the prism. However, for larger distances, the field picture is simplified because of diffraction spreading of the field.

\section{ACKNOWLEDGMENTS}

This work was supported by the Russian Science Foundation (Grant No. 18-72-10137).

[1] V. P. Zrelov, Vavilov-Cherenkov Radiation in High-Energy Physics (Israel Program for Scientific Translations, Jerusalem, 1970).

[2] A. P. Potylitsyn, Y. A. Popov, L. G. Sukhikh, G. A. Naumenko, and M. V. Shevelev, Investigation of coherent Čerenkov radiation generated by $6.1 \mathrm{MeV}$ electron beam passing near the dielectric target, J. Phys. Conf. Ser. 236, 012025 (2010).

[3] M. I. Ryazanov, M. N. Strikhanov, and A. A. Tishchenko, Diffraction radiation from an inhomogeneous dielectric film on the surface of a perfect conductor, J. Exp. Theor. Phys. 99, 311 (2004).

[4] M. Shevelev, A. Konkov, and A. Aryshev, Soft-x-ray Cherenkov radiation generated by a charged particle moving near a finite-size screen, Phys. Rev. A 92, 053851 (2015).

[5] R. Kieffer, L. Bartnik, M. Bergamaschi, V. V. Bleko, M. Billing, L. Bobb, J. Conway, M. Forster, P. Karataev, A. S. Konkov, R. O. Jones, T. Lefevre, J. S. Markova, S. Mazzoni, Y. Padilla Fuentes, A. P. Potylitsyn, J. Shanks, and S. Wang, Direct Observation of Incoherent Cherenkov Diffraction Radiation in the Visible Range, Phys. Rev. Lett. 121, 054802 (2018).

[6] E. S. Belonogaya, A. V. Tyukhtin, and S. N. Galyamin, Approximate method for calculating the radiation from a moving charge in the presence of a complex object, Phys. Rev. E 87, 043201 (2013).

[7] E. S. Belonogaya, S. N. Galyamin, and A. V. Tyukhtin, Short-wavelength radiation of a charge moving in the 
presence of a dielectric prism, J. Opt. Soc. Am. B 32, 649 (2015).

[8] S. N. Galyamin and A. V. Tyukhtin, Dielectric Concentrator for Cherenkov Radiation, Phys. Rev. Lett. 113, 064802 (2014).

[9] S. N. Galyamin, A. V. Tyukhtin, S. Antipov, and S. S. Baturin, Terahertz radiation from an ultra-relativistic charge exiting the open end of a waveguide with a dielectric layer, Opt. Express 22, 8902 (2014).

[10] A. V. Tyukhtin, V. V. Vorobev, E. S. Belonogaya, and S. N. Galyamin, Radiation of a charge in presence of a dielectric object: Aperture method, J. Instrum. 13, C02033 (2018).

[11] S. N. Galyamin, A. V. Tyukhtin, and V. V. Vorobev, Focusing the Cherenkov radiation using dielectric concentrator: simulations and comparison with theory, J. Instrum. 13, C02029 (2018).

[12] A. Fradin, Microwave Antennas (Pergamon, New York, 1961).

[13] B. M. Bolotovskii, Theory of cerenkov radiation (III), Sov. Phys. Usp. 4, 781 (1962). 